\title{
PREVALÊNCIA E FATORES ASSOCIADOS À EXPERIMENTAÇÃO DO CIGARRO ENTRE ESCOLARES DE UMA CIDADE DO INTERIOR DE MINAS GERAIS
}

\author{
PREVALENCE AND RISK FACTORS ASSOCIATED TO THE EXPERIMENTATION OF \\ CIGARETTES AMONG STUDENTS FROM A CITY OF MINAS GERAIS
}

Bianca Lisa de Faria', Bárbara Lins Silva', Clara Machado Rodrigues'ㄹ, Isabel Lucas Batista Baía', Isabele Santos Piuzana Barbosa', Natália Silveira Cruz', Raisa Furfuro e Sá', Ricardo Henrique Silva Miranda', Thauler Alves De Oliveira', Túlio Henrique Da Cruz', Olber Moreira de Faria', Siura Aparecida Borges Silva ${ }^{1,2}$

\footnotetext{
${ }^{1}$ Faculdade de Medicina da Universidade de Itaúna, Itaúna - MG

${ }^{2}$ Hospital Maternidade Odete Valadares, Belo Horizonte-MG
}

\section{Resumo}

INTRODUÇÃO: A adolescência é um momento de importantes transformações biopsicossociais, sendo a fase da vida em que há maior exposição a fatores de risco para a saúde, como o tabaco. OBJETIVO: Estimar a prevalência e fatores associados ao tabagismo entre escolares do município de ItaúnaMG. MÉTODOS: A amostra compõe-se de 340 adolescentes, sendo incluídos estudantes matriculados no ensino médio, entre 14 e 20 anos. Foi aplicado um questionário adaptado do "VI Levantamento Nacional sobre o Consumo de Drogas Psicotrópicas entre Estudantes do Ensino Fundamental e Médio das Redes Pública e Privada de Ensino nas 27 Capitais Brasileiras". A amostra simples foi selecionada através de sorteio simples. RESULTADOS: A prevalência de experimentação de tabaco do grupo avaliado foi de $28,24 \%$. Aproximadamente $60 \%$ dos que se lembram, experimentaram tabaco entre os 14 e 16 anos. Fatores como a própria curiosidade e influência de amigos foram citados como os maiores motivadores para o primeiro contato com o cigarro. $O$ fato de $o$ estudante trabalhar ou já ter trabalhado apresentou significância estatística como fator de risco para a experimentação de tabagismo na amostra avaliada. CONCLUSÃO: A prevalência de experimentação de tabaco entre os adolescentes concorda com a tendência nacional. Nesse sentido, os dados do estudo apresentado podem ser utilizados como base para planejamento de estratégias de prevenção ao tabagismo.

Palavras-chave Tabagismo, Adolescente, Comportamento do Adolescente

\section{Abstract}

INTRODUCTION: The adolescent is bound to biopsychosocial changes, and it is very likely to be exposed to risk factors, as tobacco use. OBJECTIVE: To estimate the prevalence and smoke-related risks among students from Itaúna-MG. METHODS: The sample was composed by 340 adolescents from high school, between 14 and 20 years old. We have applied an adapted version of the questionnaire from the "VI National Survey on the Use of Psychotropic Drugs among Elementary and Middle School Students of Public and Private Education Networks in the 27 Brazilian Capitals". Our sample was selected by random allocation. RESULTS: The prevalence of experimentation of tobacco was of $28,24 \%$. The average age of the first contact with tobacco was between 14 and 16 years old, according to $60 \%$ of the students who remembered the occasion. Factors as the curiosity and influence from friends were the most important risks to the first use of cigarettes. The relation between working as a risk factor for smoking was significant in our sample. CONCLUSION: The prevalence of tobacco experimentation among adolescents in our sample was similar to the national data. Our study found important information which is able to promote public health plans in order to prevent the smoking exposure.

Keywords Tobacco Use Disorder, Adolescent, Adolescent Behavior

Autora correspondente - Bianca Lisa De Faria E-mail: biancalisadefaria@outlook.com Endereço: Rua Monteiro Lobato 681, Bairro Bela Vista, Divinópolis-MG - Telefone: (37) 98816-8767 


\section{INTRODUÇÃO}

O tabagismo é considerado a principal causa de morte evitável no mundo. Cerca de $80 \%$ dos adultos fumantes declaram ter iniciado o vício antes dos dezoito anos de idade. Quanto mais precoce o início, mais intensa será a dependência e mais graves serão os danos à saúde, uma vez que o tabagismo reduz a sobrevida de maneira dose-dependente. Enquanto $80 \%$ dos não-fumantes estão vivos aos 70 anos, somente $50 \%$ dos fumantes chegam a essa idade.

A adolescência é um momento de importantes transformações biopsicossociais, em que o individuo busca por novas experiências. Consequentemente, é a fase da vida em que há maior exposição a fatores de risco para a saúde, como o tabaco.

Segundo a Organização Mundial de Saúde, por dia, aproximadamente 100 mil crianças e adolescentes tornam-se fumantes regulares em todo o mundo. No Brasil, a taxa de experimentação do tabaco entre os escolares na faixa etária de 16 a 17 anos ultrapassa $29,0 \%$, e o uso de outros métodos de consumo do tabaco, como o narguilé, tem se popularizado junto aos jovens .

Tendo em vista o impacto do consumo do cigarro e similares, seja no presente, seja no futuro dos adolescentes, identificar a prevalência e os fatores que influenciam o uso do tabaco é fundamental para, além de dimensionar o problema, servir de base para a elaboração de estratégias de prevenção. Apesar disso, as publicações científicas sobre essa temática são poucas e inconclusivas. Sendo assim, o objetivo deste estudo foi estabelecer a prevalência e os fatores associados ao tabagismo entre escolares do município de Itaúna-MG.

Material e Métodos

Itaúna é um município brasileiro do Estado de Minas Gerais, localizado a $76 \mathrm{~km}$ da capital, com uma população média de 85.000 mil habitantes. 0 presente estudo realizou-se no período de outubro a dezembro de 2016, com delineamento epidemiológico transversal, nos colégios da cidade. Foram incluídas todas as escolas públicas da cidade que ofereciam os três níveis do ensino médio $(1 \circ$, 2 은 e 3a anos), totalizando dez instituições.

Calculou-se uma amostra representativa dos alunos matriculados utilizando o método da amostragem aleatória simples, sem reposição com população finita, considerando um nível de

\begin{tabular}{|c|c|c|c|c|c|c|c|c|c|c|}
\hline $\begin{array}{l}\text { Amostras dos } \\
\text { estratos para } \\
\text { erro } 0,05\end{array}$ & & & & & & & & & & \\
\hline \multirow[t]{3}{*}{ Escola } & \multicolumn{3}{|l|}{ Manhä } & \multicolumn{3}{|c|}{ Tarde } & \multicolumn{3}{|c|}{ Noite } & \multirow[t]{3}{*}{ Tota } \\
\hline & $1^{\circ}$ Ano & $2^{\circ}$ & $3^{\circ}$ & $1^{0}$ & $2^{\circ}$ & $3^{\circ}$ & $1^{\circ}$ & $2^{\circ}$ & $3^{\circ}$ & \\
\hline & & ano & Ano & Ano & Ano & Ano & Ano & Ano & Ano & \\
\hline Escola 1 & 6 & 3 & 2 & - & - & - & . & - & - & 11 \\
\hline Escola 2 & 0 & 25 & 30 & 21 & - & - & . & 5 & 5 & 86 \\
\hline Escola 3 & 14 & 10 & 6 & - & • & - & - & • & • & 30 \\
\hline Escola 4 & 9 & 8 & 7 & . & - & - & . & - & - & 24 \\
\hline Escola 5 & 19 & 18 & 13 & - & - & - & - & - & - & 50 \\
\hline Escola 6 & 10 & 9 & 6 & * & • & $\cdot$ & * & * & • & 25 \\
\hline Escola 7 & 17 & 8 & 5 & - & - & - & - & - & - & 30 \\
\hline Escola 8* & 18 & 14 & 9 & • & • & • & * & 5 & 5 & 51 \\
\hline Escola 9 & 6 & 3 & 2 & - & - & - & . & - & - & 11 \\
\hline Escola 10 & 6 & 3 & 5 & - & - & - & 2 & 3 & 3 & 22 \\
\hline Total & 105 & 101 & 85 & 21 & 0 & 0 & 2 & 13 & 13 & 340 \\
\hline
\end{tabular}

confiança de $95 \%$ e faixa de erro de $\pm 5 \%$, o que resultou em 340 alunos. Após essa definição foi feita a estratificação da amostra, utilizando como fatores amostrais escola, turno e ano do ensino médio (TABELA 1).

A coleta de dados foi realizada por meio de um questionário adaptado do utilizado durante o "VI Levantamento Nacional sobre o Consumo de Drogas Psicotrópicas entre Estudantes do Ensino Fundamental e Médio das Redes Pública e Privada de Ensino nas 27 Capitais Brasileiras" , que possuía, além de perguntas diretamente relacionadas ao tabagismo, questões socioeconômicas.

O questionário foi aplicado dentro da sala de aula e os discentes participantes selecionados por sorteio simples pelo número de chamada. Critérios de inclusão:

- Estudantes matriculados no ensino médio das escolas públicas da cidade e residentes em Itaúna;

- Idade entre 14 e 20 anos;

- Termo de consentimento livre e esclarecido (TCLE) devidamente assinado.

Destes, foram excluídos os que se recusaram a participar. Essa faixa etária foi escolhida por contemplar aquela mais exposta ao início do tabagismo.

Os dados foram compilados no programa Microsoft Office Excel $2007^{\circledR}$. Quanto à análise dos dados, processou-se a análise univariada, através do cálculo da razão de prevalência (RP) como medida de associação entre a variável dependente (experimentação do cigarro) e as variáveis independentes sexo, conhecimento sobre os 
malefícios do cigarro e tabagismo entre pais e irmãos, sendo que em relação ao uso de outros produtos do tabaco, especificamente o narguilé, considerou-se a sua experimentação como a variável dependente e o uso do cigarro como variável independente no cálculo da RP. Realizou-se também a análise multivariada com o modelo de regressão logística, considerando a variável "Você já fumou cigarro?" como resposta e as seguintes variáveis como explicativas: "Você trabalha ou já trabalhou?", "Seus pais fumam?", "Seus irmãos fumam?", "Você já recebeu informações sobre os malefícios do cigarro em sua escola?", "Qual a escolaridade de seu pai?", "Qual a escolaridade de sua mãe", "Qual a renda mensal de sua família?", "Qual sua renda pessoal?". O objetivo foi modelar o hábito de fumar cigarro com questões socioeconômicas e, para a seleção das variáveis, foi utilizado dois métodos: o "Stepwise", com a seleção via AIC e o método "Forward" utilizando o teste de razão de verossimilhança.

Este estudo foi aprovado pelo Comitê de Ética em Pesquisa Envolvendo Seres Humanos da Universidade de Itaúna, sob CAAE no 61101816.0 .0000 .5144 .

Tabela 2 - Caracterização da amostra em relação ao sexo e idade e prevalência do uso do cigarro e narguilé

\begin{tabular}{ll}
\hline VARIÁVEIS & VALORES ABSOLUTOS(\%) \\
\hline N=340 & \\
Sexo & \\
Masculino & $170(50)$ \\
Feminino & $166(48,82)$ \\
Não responderam & $4(1,18)$ \\
Idade (anos) & $39(11,47)$ \\
$\mathbf{1 5}$ & $110(32,35)$ \\
$\mathbf{1 6}$ & $106(31,18)$ \\
$\mathbf{1 7}$ & $69(20,29)$ \\
$\mathbf{1 8}$ & $11(3,24)$ \\
$\mathbf{1 9}$ & $1(0,29)$ \\
$\mathbf{2 0}$ & $4(1,18)$ \\
Não responderam & \\
Experimentaram tabaco & $96(28,23)$ \\
Sim & $240(70,59)$ \\
Não & $4(1,18)$ \\
Não responderam & \\
Experimentaram narguilé & $51(15)$ \\
Sim & $285(83,82)$ \\
Não & $4(1,18)$ \\
Não responderam & \\
\hline
\end{tabular}

\section{RESULTADOS}

Responderam ao questionário 340 estudantes, $50,6 \%$ dos participantes eram do sexo masculino e a idade variou de 15 a 20 anos, com média de 16,7 anos (TABELA 2). Em relação aos aspectos sociais (TABELA 3 ), observa-se que $7 \%$ dos pais dos entrevistados concluíram o ensino superior e apenas 1,2\% não têm nenhuma formação escolar, enquanto a maioria das mães concluiu o ensino médio e $9,8 \%$ concluíram o ensino superior. Quanto aos aspectos econômicos (TABELA 3), nota-se que a maior parte das famílias possuía renda de até 3 salários mínimos e 59,8\% dos alunos disseram trabalhar atualmente ou já ter trabalhado.

Dos participantes, $28,6 \%$ responderam ter experimentado cigarro no decorrer de suas vidas. A idade em que houve o primeiro contato variou de 11 a 20 anos, sendo que a grande maioria $(68,25 \%)$ experimentou entre os 14 e 16 anos. Desses indivíduos, $62 \%$ eram do sexo masculino (RP: 1,58 ; IC de 95\%: 1,12 - 2,25). Analisando o período noturno de maneira isolada, a prevalência da experimentação do tabaco foi de $35,7 \%$.

Quando questionados a respeito do local da experimentação, $40,62 \%$ dos entrevistados responderam ter sido junto aos amigos, $33,3 \%$ em festas, $9,37 \%$ em casa, $7,3 \%$ na escola e $4,17 \%$ na rua enquanto sozinhos. Do total, $68,75 \%$ acreditam que a curiosidade foi o principal responsável pela experimentação; $16,7 \%$ por influência de amigos fumantes; 4,2\% por influência da família e 1,1\% por influência da mídia.

Daqueles que já tiveram contato com o tabaco, $66 \%$ declararam ter usado cigarro também durante $o$ ano de realização desta pesquisa. Acerca da freqüência do uso, 56,6\% afirmaram usar esporadicamente, $4,1 \%$ mensalmente, $5,2 \%$ semanalmente, $17,5 \%$ diariamente e $16,6 \%$ não responderam à pergunta.

Em relação ao entendimento dos adolescentes acerca dos malefícios do uso do cigarro, quando indagados sobre os riscos que eles acreditam correr um jovem que fuma cigarro às vezes, $78 \%$ dos discentes acreditam que o risco é moderado/grave, $16,4 \%$ risco leve/sem risco e $6 \%$ não responderam à pergunta. Entre os alunos que acreditam que o risco é leve/sem risco, $50 \%$ já experimentaram cigarro (RP: 1,94; IC de 95\%: 1,39 - 2,74). Em contrapartida, aproximadamente $77,6 \%$ dos estudantes disseram já ter recebido informações sobre os prejuízos do tabaco em suas escolas.

Quando abordados sobre o uso do cigarro pelos pais, $24 \%$ dos alunos afirmaram que os pais fumam, sendo que $33 \%$ desses jovens já experimentaram cigarro (RP: 1,2; IC de 95\%: 0,851,76). Em relação aos irmãos, $15 \%$ dos participantes 
Tabela 3 - Distribuição comparativa dos critérios socioeconômicos entre aqueles que ja experimentaram cigarro e 05 que nunca usaram.

\begin{tabular}{lll}
\hline VARIÁVEIS & $\begin{array}{l}\text { VALOR ABSOLUTO (\%): } \\
\text { EXPERIMENTARAM } \\
\text { TABACO (N=96) * }\end{array}$ & $\begin{array}{l}\text { VALOR ABSOLUTO (\%): } \\
\text { NĀO EXPERIMENTARAM } \\
\text { TABACO }(\mathbf{N}=240){ }^{*}\end{array}$ \\
\hline $\begin{array}{ll}\text { Você já trabalhou? } \\
\text { Sim }\end{array}$ & $66(68,75)$ & $135(56,25)$ \\
Não & $24(25)$ & $98(40,83)$ \\
$\begin{array}{l}\text { Não } \\
\text { respondeu } \\
\text { Total }\end{array}$ & $6(6,25)$ & $7(2,92)$ \\
& 96 & $\mathbf{2 4 0}$
\end{tabular}

Total 240

Renda do grupo familiar (salários mínimos)

Nenhuma $\quad 5(5,21)$

$\begin{array}{lll}\text { renda } & 10(10,42) & 34(14,17)\end{array}$

$\begin{array}{lll}<1 & 10(10,42) & 34(14,17) \\ 1 \text { a 3 } & 43(44,79) & 128(53,33)\end{array}$

6 a $9 \quad 6(6,25) \quad 12(5)$

9 a $12 \quad 1(1,04) \quad 1(0,42)$

12 a $15 \quad 2(2,08) \quad 0(0)$

$>15 \quad 0(0) \quad 1(0,42)$

Não $\quad 2(2,08) \quad 10(4,16)$

$\begin{array}{lll}\text { respondeu } & 96 & 240 \\ \text { Total } & 96\end{array}$

Escolaridade do pai

Não estudou $3(3,12) \quad 1(0,42)$

Primeira à $\quad 21(21,87) \quad 50(20,82)$

$\begin{array}{ll}\text { quarta série } & 18(18,75)\end{array}$

Quinta à $\quad 18(18,75) \quad 49(20,42)$

Ensino médio $28(29,17) \quad 75(31,25)$

Ensino $6(6,25) \quad 18(7,5)$

superior
Especialização $4(4,17) \quad 7(2,92)$

Não sabe $\quad 16(16,67) \quad 39(16,25)$

\begin{tabular}{|c|c|c|}
\hline $\begin{array}{l}\text { Não } \\
\text { respondeu } \\
\text { Total }\end{array}$ & $\begin{array}{l}0(0) \\
96\end{array}$ & $\begin{array}{l}1(0,42) \\
240\end{array}$ \\
\hline \multicolumn{3}{|c|}{ Escolaridade da mãe } \\
\hline Não estudou & $2(2,08)$ & $1(0,42)$ \\
\hline $\begin{array}{l}\text { Primeira à } \\
\text { quarta série }\end{array}$ & $13(13,54)$ & $40(16,66)$ \\
\hline $\begin{array}{l}\text { Quinta à } \\
\text { oitava série }\end{array}$ & $27(28,12)$ & $54(22.5)$ \\
\hline Ensino médio & $34(35,42)$ & $81(33,75)$ \\
\hline $\begin{array}{l}\text { Ensino } \\
\text { superior }\end{array}$ & $6(6,25)$ & $27(11,25)$ \\
\hline Especialização & $4(4,17)$ & $9(3,75)$ \\
\hline Não sabe & $10(10,42)$ & $27(11,25)$ \\
\hline $\begin{array}{l}\text { Não } \\
\text { respondeu }\end{array}$ & $0(0)$ & $1(0,42)$ \\
\hline Total & 96 & 240 \\
\hline \multicolumn{3}{|c|}{ Uso de tabaco pelos pais } \\
\hline $\begin{array}{l}\text { Nenhum dos } \\
\text { dois usa }\end{array}$ & $69(71,87)$ & $186(77,5)$ \\
\hline $\begin{array}{l}\text { Somente a } \\
\text { mãe }\end{array}$ & $7(7,29)$ & $18(7,5)$ \\
\hline Somente o pai & $16(16,67)$ & $27(11,25)$ \\
\hline Ambos usam & $3(3,13)$ & $9(3,75)$ \\
\hline $\begin{array}{l}\text { Não } \\
\text { responderam }\end{array}$ & $1(1,04)$ & $0(0)$ \\
\hline Total & 96 & 240 \\
\hline \multicolumn{3}{|c|}{ Uso de tabaco por irmãos } \\
\hline $\begin{array}{l}\text { Não tenho } \\
\text { irmãos }\end{array}$ & $11(11,46)$ & $33(13,75)$ \\
\hline Sim & $18(18,75)$ & $26(10,83)$ \\
\hline Não & $67(69,79)$ & $180(75)$ \\
\hline $\begin{array}{l}\text { Não } \\
\text { responderam }\end{array}$ & $0(0)$ & $1(0,42)$ \\
\hline Total & 96 & 240 \\
\hline
\end{tabular}

afirmaram que possuem irmãos fumantes e, desses, 40,9\% já fumaram (RP: 1,49; IC de 95\%: 0,99-2,25).

Quanto ao uso do narguilé, $15,1 \%$ dos jovens já utilizaram o produto. A maioria deles $(60,8 \%)$ era do sexo masculino e $68,6 \%$ já fumaram cigarro (RP de 5,2; 95\% Cl: 3,03-8,96).

$\mathrm{Na}$ análise multivariada a variável que se mostrou relacionada com a experimentação do tabaco foi trabalhar ainda na adolescência (OR 2,32 IC:95\% $p<0,01)$.

Discussão

Por tratar-se de uma pesquisa realizada com estudantes do ensino médio a maior parte da amostra possuía entre 16-17 anos. A taxa geral de experimentação do cigarro foi de $28,6 \%$, resultado próximo ao obtido no estudo PeNSE 2015, realizado com 109104 estudantes brasileiros, em que a faixa etária de 16-17 anos apresentou percentual de experimentação do produto de $29 \%$. Como esperado, a prevalência foi maior entre os alunos do período noturno, pois os comportamentos de risco à saúde são, de maneira geral, consideravelmente maiores entre esse público .

O primeiro contato com o cigarro teve seu pico entre os 14-16 anos. Esse achado esta em consonância com praticamente todas as publicações nacionais similares, reafirmado o inicio precoce do hábito de fumar.

Cerca de $26 \%$ dos usuários afirmaram fazer uso regular (mensal, semanal ou diário) da substância, sendo portanto susceptíveis à dependência da nicotina e de todos os seus demais efeitos deletérios. Trata-se de um valor considerável, principalmente porque $16,6 \%$ dos alunos que já fumaram não responderam sobre a freqüência do uso, o que pode subestimar os resultados sobre a regularidade do consumo do cigarro e consequente risco de se tornarem fumantes regulares.

Os principais fatores associados à experimentação do cigarro durante a adolescência citados na literatura são: sexo masculino, nível socioeconômico baixo, o fato de trabalhar já na adolescência e presença de tabagismo entre pais, irmãos e amigos .

Neste estudo o sexo masculino associou-se com a maior experimentação do tabaco, como observado em outras pesquisas similares. No entanto, a diferença de prevalência foi muito sutil sendo esse dado bastante variável entre os trabalhos sobre o tema, não havendo ainda consistência 
quanto à diferença de prevalência entre os sexos .

Apesar de o baixo nível socioeconômico ser apontado como um fator para o contato com o tabaco, existem poucas publicações sobre o tema e os dados a esse respeito na literatura são bastante divergentes. As principais explicações pautadas para essa associação seriam a menor acessibilidade aos serviços de saúde, estresse psicológico, piores condições de vida e menor acesso a informação pelos estratos sociais mais baixos. Pela análise multivariada não se observou correlação entre a renda familiar e escolaridade dos pais e o tabagismo entre os alunos, assim como em outros estudos sobre o tema .

Por sua vez, o fato do estudante trabalhar ou já ter trabalhado durante a vida, foi o único indicador de condição socioeconômica que se configurou como um importante fator de risco para o contato com o tabaco, como verificado também em outros artigos. Várias são as hipóteses para explicar essa associação, como o adolescente possuir renda suficiente para comprar o produto, estresse no trabalho, assim como uma possível transição precoce para a vida adulta . Cabe ressaltar que, na análise multivariada, o adolescente possuir renda pessoal, por si só, não se configurou um fator de risco independente.

Em relação ao tabagismo de pais e irmãos, embora a razão de prevalência encontrada na analise univariada aponte para uma possível associação, o intervalo de confiança obtido não nos permite excluir a hipótese nula. Ainda que sejam variáveis frequentemente apontados como fatores de risco, nem todas as publicações, especialmente as mais recentes, confirmam essa relação indicando que, na atualidade, o estimulo para o contato com o tabaco esta comumente fora do núcleo familiar.

Em relação aos amigos fumantes, existem evidências de que se trata de um dos mais importantes fatores de risco para a iniciação ao tabagismo, verificando que adolescentes não fumantes que têm amigos que o fazem possuem chances maiores de, no futuro, começar a fumar-. Neste estudo, aproximadamente $17 \%$ dos estudantes que já fumaram disseram que o fizeram devido a essa influência. O principal fator que levou a experimentação do cigarro foi curiosidade, uma característica intrínseca do ser humano que em alguns períodos da vida torna-se ainda mais acentuada, como na adolescência, em que os indivíduos estão dispostos a novas vivências. Notase que, dentre as variáveis estudadas, a mídia foi a menos referida pelos estudantes como a responsável por seu primeiro contato com o cigarro. E $s$ s e achado reflete a mudança radical no perfil da publicidade envolvendo a indústria do tabaco no Brasil nos últimos anos. No passado, a mídia era a principal responsável pela indução ao consumo do cigarro pela população, retratando seu uso como sinônimo de sucesso e irreverência. A proibição dessas propagandas no país, assim como a divulgação em massa de seus malefícios, tem papel importante na redução da prevalência do tabagismo que vêm sendo observada no Brasil . Contudo, os efeitos da mídia "indireta", como espetáculos e clipes musicais, são difíceis de mensurar e podem ser responsáveis por parcela da curiosidade que os jovens possuem de usar a substância.

Cabe salientar que apesar de o narguilé equivaler a 100 cigarros, há uma desinformação da população geral sobre os seus danos. Em torno de $70 \%$ dos participantes que já fumaram cigarro também utilizaram narguilé. Nesse aspecto, já ter experimentado cigarro configurou-se um importante fator associado ao uso de narguilé, demonstrando que ele pode atuar como porta de entrada para outros derivados do tabaco ainda mais nocivos.

Ademais, é importante destacar que se notou uma associação entre a experimentação do cigarro e a crença de que os riscos que um jovem que fuma esta exposto são leves ou ausentes. Em contrapartida, verificou-se que apenas $22 \%$ dos entrevistados nunca receberam informações sobre os danos do cigarro em suas escolas. Assim sendo, é importante repensar se esse conhecimento esta sendo passado de maneira clara para os estudantes, uma vez que estudos já demonstraram que abordaro uso de drogas como o tabaco de maneira isolada com os alunos é, na verdade, contraproducente, podendo aumentar o consumo dessa substância entre eles

Entre as limitações deste estudo, ressalta-se o fato de a pesquisa ter sido realizada somente em escolas públicas e com alunos do ensino médio, portanto, outros resultados poderiam ser encontrados caso fossem incluídas escolas privadase alunos de outras faixas etárias. Em adição, no delineamento transversal o viés da causalidade reversa não pode ser eliminado, pois fatores de risco e desfecho são vistos no mesmo momento. 


\section{CONCLUSÃO}

Entre os adolescentes matriculados em escolas públicas de Itaúna, foi estimada uma prevalência de experimentação de tabaco e narguilé de $28,24 \%$ e $15 \%$, respectivamente. Os dados são comparáveis aos grandes estudos nacionais já realizados. Na população em análise, o trabalho demonstrou-se como fator de risco para o uso de tabaco, de forma substancialmente significativa. Observa-se que a primeira experimentação ocorre geralmente entre 14 e 16 anos, sendo motivada, principalmente, pela própria curiosidade ou influência de amigos.

Percebe-se impacto importante exercido por medidas legais nas últimas décadas, no sentido da restrição à propaganda relacionada aos produtos derivados do tabaco, tendo em vista que "mídia" foi a influência menos citada pelos adolescentes que já experimentaram tabaco.

O presente trabalho, em seu microambiente, configura-se como uma boa ferramenta para embasar políticas municipais na prevenção do tabagismo entre adolescentes, uma vez que levantou os principais parâmetros relacionados à experimentação do mesmo. Não obstante, existe necessidade de desenvolvimento de novos estudos que possam avaliar de forma mais efetiva as variáveis socioeconômicas que competem na experimentação de tabaco. Dessa forma, ações em saúde coletiva podem ser mais assertivas e específicas no combate da primeira exposição ao tabaco e seus derivados entre os jovens.

\section{Referência}

1. Samet JM. Tobacco Smoking: The Leading Cause of Preventable Disease Worldwide. Thorac Surg Clin 2013;23(2):10312.

2. Andrade APA de, Bernardo ACC, Viegas CA de A, Ferreira DBL, Gomes TC, Sales MR. Prevalência e características do tabagismo em jovens da Universidade de Brasília. J Bras Pneumol 2006;32(1):23-8.

3. Vitória PD, Silva SA, Vries HD. Avaliação longitudinal de programa de prevenção do tabagismo para adolescentes. Rev Saúde Pública 2011;45(2):344-54.

4. Kumar V, Abbas AK, Aster JC. Robbins and Cotran Pathologic Basis of Disease. 9th Editio. Philadelphia: Elsevier Saunders; 2015.

5. Zappe JG, Dell'Aglio DD. Adolescência em diferentes contextos de desenvolvimento: risco e proteção em uma perspectiva longitudinal. Psico 2016;47(2):99-110.

6. Warren CW, Riley L, Asma S, et al. Tobacco use by youth: a surveillance report from the Global Youth Tobacco Survey project. Bull World Health Organ 2000;78:868-76.

7. Instituto Brasileiro de Geografia e Estatística, editor. Pesquisa nacional de saúde do escolar, 2015. Rio de Janeiro: IBGE; 2016.

8. Barreto SM, Giatti L, Oliveira-Campos M, Andreazzi MA, Malta DC. Experimentation and use of cigarette and other tobacco products among adolescents in the Brazilian state capitals (PeNSE 2012). Rev Bras Epidemiol 2014;17(suppl 1):62-76.

9. Oliveira HF, Martins LC, Lígia de Fátima NR, Akerman M. Fatores de risco para uso do tabaco em adolescentes de duas escolas do município de Santo André, São Paulo. Rev Paul Pediatr 2010;28(2):200-7.

10. Cidades I. Censo 2010. Ww Cid Ibge Gov Br Acesso Em Novembro De 2016;

11. Carlini, E. A. VI Levantamento Nacional sobre o Consumo de Drogas Psicotrópicas entre Estudantes do Ensino Fundamental e Médio das Redes Pública e Privada de Ensino nas 27 Capitais Brasileiras. São Paulo: Centro Brasileiro de Informações sobre Drogas Psicotrópicas; 2010.

12. Farias Júnior JC de, Nahas MV, Barros MVG de, et al. Comportamentos de risco à saúde em adolescentes no Sul do Brasil: prevalência e fatores associados. 2009;

13. Menezes AHR, Dalmas JC, Scarinci IC, Maciel SM, Cardelli AAM. Fatores associados ao uso regular de cigarros por adolescentes estudantes de escolas públicas de Londrina, Paraná, Brasil. Cad Saúde Pública 2014;30(4):774-84.

14. Figueiredo VC, Szklo AS, Costa LC, et al. ERICA: smoking prevalence in Brazilian adolescents. Rev Saúde Pública [Internet] 2016 [cited 2019 Jan 8];50(suppl 1). Available from:

http://www.scielo.br/scielo.php?script=sci_arttext\&pid=S0034-89102016000200313\&lng=en\&tlng=en

15. Malcon MC, Menezes AMB, Maia M de FS, Chatkin M, Victora CG. Prevalência e fatores de risco para tabagismo em adolescentes na América do Sul: uma revisão sistemática da literatura. Rev Panam Salud Pública 2003;13(4):222-8. 
16. Vargas LS, Lucchese R, Silva AC da, Guimarães RA, Vera I, Castro PA de. Determinants of tobacco use by students. Rev Saúde Pública [Internet] 2017 [cited 2019 Jan 8];51(0). Available from:

http://www.scielo.br/scielo.php?script=sci_arttext\&pid=S0034-89102017000100233\&lng=en\&tlng=en

17. Cristina A, Alves J, Perelman J. Desigualdades socioeconómicas no tabagismo em jovens dos 15 aos 17 anos. Rev Port Saúde Pública 2016;34(1):69-76.

18. Finkelstein DM, Kubzansky LD, Goodman E. Social status, stress, and adolescent smoking. J Adolesc Health 2006;39(5):678-85.

19. Souza DPO de, Silveira Filho DX da. Uso recente de álcool, tabaco e outras drogas entre estudantes adolescentes trabalhadores e não trabalhadores. Rev Bras Epidemiol 2007;10(2):276-87.

20. Hallal AL de LC, Figueiredo VC, Moura L de, Prado RR do, Malta DC. Uso de outros produtos do tabaco entre escolares brasileiros (PeNSE 2012). Cad Saúde Pública [Internet] 2017 [cited 2019 Jan 8];33(suppl 3). Available from:

http://www.scielo.br/scielo.php?script=sci_arttext\&pid=S0102-311X2017001505009\&lng=pt\&tlng=pt

21. Urrutia-Pereira M, Oliano VJ, Aranda CS, Mallol J, Solé D. Prevalence and factors associated with smoking among adolescents. J Pediatr (Rio J) 2017;93(3):230-7.

22. Bricker JB, Peterson A V, Andersen MR, Rajan KB, Leroux BG, Sarason IG. Childhood friends who smoke: do they influence adolescents to make smoking transitions? Addict Behav 2006;31(5):889-900.

23. Faria BL, Brieske CM, Cosgarea I, et al. A smoking prevention photoageing intervention for secondary schools in Brazil delivered by medical students: protocol for a randomised trial. BMJ Open 2017;7(12):e018589.

24. da Silva Grossi FR, de Araújo FRF, Rêgo NM, dos Santos Souza R. Fatores influenciadores e às consequências sobre o uso do tabaco na adolescência: uma revisão sistemática. Rev Ciênc SAÚDE OESTE BAIANO 2017;2(1).

25. Ribeiro DCB, Ewald AP. Ética e publicidade infantil. Comun Inov 2010;11(20).

26. de Assis Viegas CA. Formas não habituais de uso do tabaco. J Bras Pneumol 2008;34(12):1069-73.

27. do Nascimento MO, De Micheli D. Avaliação de diferentes modalidades de ações preventivas na redução do consumo de substâncias psicotrópicas em estudantes no ambiente escolar: um estudo randomizado. Ciênc Saúde Coletiva 2015;20(8):2499-510. 